\title{
Straight-Line Orthogonal Drawings of Binary and Ternary Trees ${ }^{\star}$
}

\author{
Fabrizio Frati \\ Dipartimento di Informatica e Automazione, Università di Roma Tre \\ frati@dia.uniroma3.it
}

\begin{abstract}
In this paper we provide upper and lower bounds on the area requirement of straight-line orthogonal drawings of $n$-node binary and ternary trees. Namely, we show algorithms for constructing orderpreserving straight-line orthogonal drawings of binary trees in $O\left(n^{1.5}\right)$ area, straight-line orthogonal drawings of ternary trees in $O\left(n^{1.631}\right)$ area, and straight-line orthogonal drawings of complete ternary trees in $O\left(n^{1.262}\right)$ area. As far as we know, the ones we present are the first algorithms achieving sub-quadratic area for these problems. Further, for upward order-preserving straight-line orthogonal drawings of binary trees and for order-preserving straight-line orthogonal drawings of ternary trees we provide $\Omega\left(n^{2}\right)$ area lower bounds, that we also prove to be tight.
\end{abstract}

\section{Introduction}

The design of algorithms for constructing orthogonal and straight-line drawings of binary and ternary trees, that are trees whose maximum degree is bounded by three and four, respectively, has attracted considerable research efforts in the Graph Drawing community. Orthogonal and straight-line planar drawings are easily readable by the viewer and hence they are among the most studied drawing standards. When dealing with orthogonal or straight-line tree drawings, it is common to consider area minimization as an important aesthetic requirement to satisfy. The study of area minimization for binary and ternary tree drawings has been motivated by VLSI circuits design and it is still attractive for the sake of rendering acyclic relationships on a screen limited by a finite resolution rule. Nevertheless, the beauty of some combinatorial and geometric open problems concerning area minimization of straight-line and orthogonal drawings of trees justifies their study even looking at them from a purely theoretical point of view.

Almost thirty years ago, Valiant proved in 12 that every $n$-node ternary tree admits a $\Theta(n)$ area orthogonal drawing. Such a result was strengthened in [5], where Dolev and Trickey proved that ternary trees admit $\Theta(n)$ area orderpreserving orthogonal drawings. A $\Theta(n \log \log n)$ optimal bound for upward orthogonal drawings of binary trees was proved by Garg et al. in [6], while in [9] Kim showed that $\Theta(n \log n)$ area is an optimal bound for upward orthogonal

\footnotetext{
* Work partially supported by MUR under Project MAINSTREAM Algorithms for Massive Information Structures and Data Streams.
} 
drawings of ternary trees. Concerning the area requirement of planar straightline drawings, Garg and Rusu proved in [8] that linear area suffices for bounded degree trees, while $\Theta(n \log n)$ area is asymptotically optimal if the drawing is required to be upward and order-preserving [7].

Drawings that are simultaneously straight-line and orthogonal provide extremely high readability of the combinatorial structure of a tree, and hence it is a serious lack in the literature that only few results concerning area minimization of straight-line orthogonal drawings of binary and ternary trees are known. Chan et al. in [1], and Shin et al. in [1] have shown that $O(n \log \log n)$ area suffices for straight-line orthogonal drawings of binary trees. Further, it has been shown in 31] that binary trees admit upward straight-line orthogonal drawings in $O(n \log n)$ area. Such an area bound is worst-case optimal, as proved in 11 .

In this paper we present the following results: (i) order-preserving straightline orthogonal drawings of binary trees can be constructed in $O\left(n^{1.5}\right)$ area (Section 13); (ii) upward order-preserving straight-line orthogonal drawings of binary trees require (and can be realized in) $\Omega\left(n^{2}\right)$ area (Section 3 ); (iii) straightline orthogonal drawings of ternary trees can be constructed in $O\left(n^{1.631}\right)$ area (Section 4); (iv) order-preserving straight-line orthogonal drawings of ternary trees require (and can be realized in) $\Omega\left(n^{2}\right)$ area (Section 4); (v) straight-line orthogonal drawings of complete ternary trees can be constructed in $O\left(n^{1.262}\right)$ area (Section 5); and (vi) there exist ternary trees for which the minimum side of any straight-line orthogonal drawing is $\Omega\left(n^{0.438}\right)$ and, for complete ternary trees, such a bound is tight (Section 5 ).

Table 1. Summary of the best known area bounds for straight-line orthogonal drawings of binary and ternary trees. For complete trees the order-preserving column is not considered, since such trees are symmetric. Straight-line orthogonal upward drawings of ternary trees cannot generally be constructed.

\begin{tabular}{|c|c|c|c|c|c|c|}
\cline { 2 - 8 } \multicolumn{1}{c|}{} & Upward & Order-preserving & Upper Bound & Ref. & Lower Bound & Ref. \\
\hline Complete Binary & $\checkmark$ & & $O(n)$ & {$[3$} & $\Omega(n)$ & trivial \\
\hline Complete Binary & & & $O(n)$ & {$[10]$} & $\Omega(n)$ & trivial \\
\hline Binary & $\checkmark$ & $\checkmark$ & $O\left(n^{2}\right)$ & {$[3]$} & $\Omega\left(n^{2}\right)$ & Th. [1 \\
\hline Binary & $\checkmark$ & & $O(n \log n)$ & {$[3]$} & $\Omega(n \log n)$ & {$[1]$} \\
\hline Binary & & $\checkmark$ & $O\left(n^{1.5}\right)$ & Th. [2 & $\Omega(n)$ & trivial \\
\hline Binary & & & $O(n \log \log n)$ & $[1] 1]$ & $\Omega(n)$ & trivial \\
\hline Complete Ternary & $\checkmark$ & & \multicolumn{4}{|c|}{ non-drawable } \\
\hline Complete Ternary & & & $O\left(n^{1.262}\right)$ & Th. [6] & $\Omega(n)$ & trivial \\
\hline Ternary & $\checkmark$ & $\checkmark$ & \multicolumn{4}{c|}{ non-drawable } \\
\hline Ternary & $\checkmark$ & & \multicolumn{4}{c|}{ non-drawable } \\
\hline Ternary & & $\checkmark$ & $O\left(n^{2}\right)$ & Th. [4 & $\Omega\left(n^{2}\right)$ & Th. [3 \\
\hline Ternary & & & $O\left(n^{1.631}\right)$ & Th. [5 & $\Omega(n)$ & trivial \\
\hline
\end{tabular}

\section{Preliminaries}

We assume familiarity with trees and their drawings (see also [4]).

A rooted tree $T$ is a tree with one distinguished node, called root and denoted by $r(T)$. In the following we assume that binary and ternary trees are rooted 
at any node of degree at most two and three, respectively. A spine in $T$ is a path connecting $r(T)$ to a leaf. A double-spine in $T$ is a path connecting two leaves and passing through $r(T)$. A tree is ordered if an order of the children of each node is specified. For an ordered binary tree we talk about left and right child. For an ordered ternary tree we talk about left, middle, and right child. The subtrees rooted at the left, middle, and right child of a node $u$ are the left, middle, and right subtree of $u$, respectively. The subtree of a given tree rooted at node $u$ is denoted by $T(u)$. Removing a path $\mathcal{P}$ from a tree disconnects the tree into connected components. The ones containing children of nodes in $\mathcal{P}$ are subtrees of $\mathcal{P}$. If the tree is ordered, then each component is a left, middle, or right subtree of $\mathcal{P}$, depending on whether the root of such subtree is a left, middle, or right child of a node in $\mathcal{P}$. We denote by $|T|$ the number of nodes in a tree $T$. The heaviest tree in a set of trees is the one with the greatest number of nodes. A complete tree is such that all non-leaf nodes have the same degree and all spines have the same number of nodes, called the height of the tree.

A straight-line orthogonal grid drawing of a binary or ternary tree is a mapping of its nodes to distinct points with integer coordinates and of its edges to horizontal or vertical segments between such points. A drawing is planar if no two segments cross, but, possibly, at common end-points. In the following we use SO-drawing as short for straight-line orthogonal planar grid drawing. An SO-drawing is upward if every node is drawn not below its children. An SOdrawing $\Gamma$ is order-preserving if, for every node $u$, the segments connecting $u$ to its left child, middle child, right child and parent appear in $\Gamma$ in this order around $u$. When we talk about order-preserving drawings, we suppose that trees are ordered. Consider an SO-drawing $\Gamma$ of a rooted tree $T$. Denote by $l$ the vertical half-line starting at $r(T)$ and directed upward. Then $\Gamma$ has the top visibility property if no node, but for $r(T)$, is placed on $l$ and no edge crosses $l$. Denote by $r$ the horizontal line through $r(T)$. Then $\Gamma$ has the side visibility property if no node, but for $r(T)$, is placed on $r$ and no edge crosses $r$. The width (height) of a drawing is the number of vertical (horizontal) grid lines intersecting it. The area of a drawing is its height multiplied by its width.

\section{Straight-Line Orthogonal Order-Preserving Drawings of Binary Trees}

First, we show that order-preserving upward SO-drawings of binary trees generally require quadratic area. Such a bound is matched by an $O\left(n^{2}\right)$ upper bound obtained by using the well-known $h$-v layout (see, e.g., [3]).

Theorem 1. There exists an $n$-node binary tree $T$ requiring $\Omega\left(n^{2}\right)$ area in any upward order-preserving SO-drawing.

Proof: Assume $n \equiv 0 \bmod 6$. Tree $T$ is composed of (see Fig. 11a): (i) an $n / 6$ node spine $C_{1}:\left(m_{0}=r(T), m_{1}, \ldots, m_{\frac{n}{6}-2}, m_{\frac{n}{6}-1}\right)$, with $m_{i}$ left child of $m_{i-1}$, for $1 \leq i \leq \frac{n}{6}-1$; (ii) an $n / 6$-node spine $C_{2}:\left(p_{0}=r(T), p_{1}, \ldots, p_{\frac{n}{6}-2}, p_{\frac{n}{6}-1}\right)$, with $p_{i}$ right child of $p_{i-1}$, for $1 \leq i \leq \frac{n}{6}-1$; (iii) the right child $m_{i}^{r}$ of each 


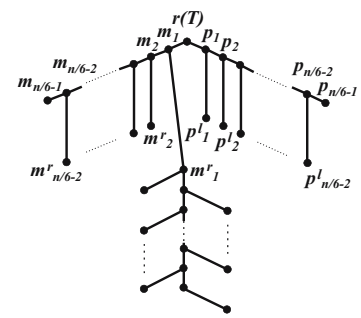

(a)

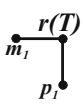

(b)

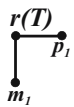

(c)

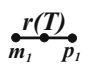

(d)

Fig. 1. (a) Tree $T$ providing the lower bound of Theorem 1 (b)-(c)-(d) Possible placements of $r(T)$ and its children.

node $m_{i}$ of $C_{1}$, with $1 \leq i \leq \frac{n}{6}-2$; (iv) the left child $p_{i}^{l}$ of each node $p_{i}$ of $C_{2}$, with $1 \leq i \leq \frac{n}{6}-2$; (v) a path $C_{3}$ of $n / 6+3$ nodes, alternating between right and left children, such that one end-vertex of $C_{3}$ is $m_{1}^{r}$; and (vi) $n / 6+3$ leaves attached to $C_{3}$, alternating between left and right children.

Consider any upward order-preserving SO-drawing $\Gamma$ of $T$. In [6] it is shown that $C_{3}$ and its attached leaves require $\Omega(n)$ height in any upward order-preserving drawing. Consider the relative position of $r(T)$ and its children in $\Gamma$. Three are the cases; either $m_{1}$ is to the left of $r(T)$ and $p_{1}$ is below $r(T)$ (see Fig. 1].b), or $m_{1}$ is below $r(T)$ and $p_{1}$ is to the right of $r(T)$ (see Fig. 1. c), or $m_{1}$ is to the left of $r(T)$ and $p_{1}$ is to the right of $r(T)$ (see Fig. 11d). Suppose $m_{1}$ is to the left of $r(T)$. We prove by induction that each node $m_{i}$ of $C_{1}$, with $1 \leq i \leq \frac{n}{6}-1$, is drawn at least one unit to the left of its parent. The claim holds in the base case by the assumption that $m_{1}$ is to the left of $m_{0}=r(T)$. If $m_{i}$ is to the left of $m_{i-1}$, then the edges from $m_{i}$ to its children are drawn towards the left and the bottom. Since the drawing is order-preserving, $m_{i}^{r}$ must be below $m_{i}$ and $m_{i+1}$ to the left of $m_{i}$. So each node $m_{i}$, with $1 \leq i \leq \frac{n}{6}-1$, is drawn at least one unit to the left of its parent, implying a linear lower bound on the width of $\Gamma$. If $m_{1}$ is not to the left of $r(T)$ then $p_{1}$ is to the right of $r(T)$ and a similar argument shows that each node $p_{i}$, with $1 \leq i \leq \frac{n}{6}-1$, is at least one unit to the right of its parent, again implying a linear lower bound on the width of $\Gamma$. Hence both the height and the width of $\Gamma$ are $\Omega(n)$.

Now we turn to non-upward drawings, showing that sub-quadratic area suffices for order-preserving SO-drawings:

Theorem 2. Any n-node binary tree $T$ admits an $O\left(n^{1.5}\right)$ area order-preserving SO-drawing.

Proof: We describe an inductive algorithm constructing an order-preserving SOdrawing $\Gamma$ of $T$ satisfying the side visibility property. If $n=1$, then $\Gamma$ is trivially constructed. Suppose $n>1$. Select a double-spine $\pi=\left(u_{k}, u_{k-1}, \ldots, u_{1}, u_{0}=\right.$ $\left.r(T)=v_{0}, v_{1}, \ldots, v_{m}\right)$ in $T$. How to choose $\pi$ is discussed later. Denote by $p_{i}$ the non-spine child of a node $u_{i} \in \pi$ and by $q_{j}$ the non-spine child of a node $v_{j} \in \pi$. 


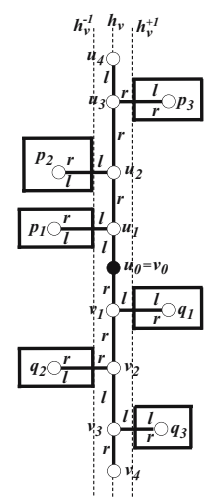

(a)

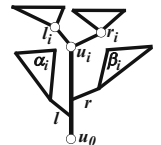

(b)

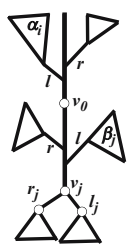

(c)

Fig. 2. Illustrations for the algorithm in the proof of Theorem 2 Left (right) edges are labeled $l(r)$. Label $l(r)$ inside a subtree shows the direction of the edge from the root to its left (right) child.

Recursively construct drawings $\Gamma\left(p_{i}\right)$ of $T\left(p_{i}\right)$ and $\Gamma\left(q_{j}\right)$ of $T\left(q_{j}\right)$ satisfying the side visibility property, for $1 \leq i<k$ and $1 \leq j<m$. Let $h_{v}, h_{v}^{-1}$, and $h_{v}^{1}$ be vertical grid lines with $h_{v}^{-1}\left(h_{v}^{1}\right)$ one unit to the left (to the right) of $h_{v}$. Draw $r(T)$ on $h_{v}$. For $i=1,2, \ldots, k-1$, if $p_{i}$ is the left child of $u_{i}$ rotate $\Gamma\left(p_{i}\right)$ of $\pi$ and place it so that the rightmost vertical line intersecting it is $h_{v}^{-1}$ and with the lowest horizontal line intersecting it one unit above the highest horizontal line intersecting $\Gamma\left(p_{i-1}\right)$ or $u_{i-1}$; otherwise $\left(p_{i}\right.$ is the right child of $\left.u_{i}\right)$, place $\Gamma\left(p_{i}\right)$ so that the leftmost vertical line intersecting it is $h_{v}^{1}$ and with the lowest horizontal line intersecting it one unit above the highest horizontal line intersecting $\Gamma\left(p_{i-1}\right)$ or $u_{i-1}$. Draw $u_{i}$ on $h_{v}$ on the same horizontal line of its already drawn child (or one unit above the highest horizontal line intersecting $\Gamma\left(p_{i-1}\right)$ or $u_{i-1}$ if no child of $u_{i}$ has been drawn). Draw $u_{k}$ on $h_{v}$ one unit above the highest horizontal line intersecting $\Gamma\left(p_{k-1}\right)$ or $u_{k-1}$. For $j=1,2, \ldots, m-1$, if $q_{j}$ is the right child of $v_{j}$ rotate $\Gamma\left(q_{j}\right)$ of $\pi$ and place it so that the rightmost vertical line intersecting it is $h_{v}^{-1}$ and with the highest horizontal line intersecting it one unit below the lowest horizontal line intersecting $\Gamma\left(q_{j-1}\right)$ or $v_{j-1}$; otherwise $\left(q_{j}\right.$ is the left child of $\left.v_{j}\right)$, place $\Gamma\left(q_{j}\right)$ so that the leftmost vertical line intersecting it is $h_{v}^{1}$ and with the highest horizontal line intersecting it one unit below the lowest horizontal line intersecting $\Gamma\left(q_{j-1}\right)$ or $v_{j-1}$. Draw $v_{j}$ on $h_{v}$ on the same horizontal line of its already drawn child (or one unit below the lowest horizontal line intersecting $\Gamma\left(q_{j-1}\right)$ or $v_{j-1}$ if no child of $v_{j}$ has been drawn). Draw $v_{m}$ on $h_{v}$ one unit below the lowest horizontal line intersecting $\Gamma\left(q_{m-1}\right)$ or $v_{m-1}$ (see Fig. 2] a).

It's easy to see that the constructed drawing $\Gamma$ is an order-preserving SOdrawing satisfying the side visibility property. Let's analyze the area requirement of $\Gamma$. Concerning its height, there is at least one node of $T$ on each horizontal grid line intersecting $\Gamma$, hence the height of $\Gamma$ is $O(n)$. Denote by $W(T)$ the width of the drawing constructed by the described algorithm when its input is binary tree $T$. Let also $W(n)=\max \{W(T)\}$ over all binary trees $T$ with $n$ 
nodes. Since all subtrees drawn to the left (to the right) of $\pi$ are aligned on their right side (on their left side) and since $W(n)$ is a non-decreasing function of $n$, then $W(n)=W\left(n_{l}\right)+W\left(n_{r}\right)+1$, where $n_{l}\left(n_{r}\right)$ is the number of nodes in the heaviest subtree drawn to the left (to the right) of $\pi$. To get a good bound for $W(n)$ we need to carefully choose $\pi$. A technique similar to the one we present was introduced in 2] for selecting (single) spines. $\pi$ is composed of two spines $\mathcal{U}=\left(u_{0}, u_{1}, \ldots, u_{k}\right)$ and $\mathcal{V}=\left(v_{0}, v_{1}, \ldots, v_{m}\right)$. Spine $\mathcal{U}$ is iteratively selected as follows: $u_{0}=r(T), u_{1}$ is the left child of $u_{0}$. Denote by $l_{i}$ and by $r_{i}$ the left and right child of $u_{i}$, respectively. Denote also by $\alpha_{i}$ and by $\beta_{i}$ the heaviest left subtree and the heaviest right subtree of path $\left(u_{1}, \ldots, u_{i-1}\right)$ (see Fig. 2] b). If $\left|\alpha_{i}\right|+\left|T\left(r_{i}\right)\right| \leq\left|\beta_{i}\right|+\left|T\left(l_{i}\right)\right|$ then set $u_{i+1}=l_{i}$, otherwise set $u_{i+1}=r_{i}$. Spine $\mathcal{V}$ is iteratively selected as follows: $v_{0}=r(T), v_{1}$ is the right child of $u_{0}$. Denote by $l_{j}$ and by $r_{j}$ the left and right child of $v_{j}$, respectively. Denote by $\alpha_{j}$ the one between the heaviest right subtree of path $\left(v_{1}, \ldots, v_{j-1}\right)$ and the heaviest left subtree of $\mathcal{U} \backslash u_{0}$ that has the greatest number of nodes. Denote also by $\beta_{j}$ the one between the heaviest left subtree of path $\left(v_{1}, \ldots, v_{j-1}\right)$ and the heaviest right subtree of $\mathcal{U} \backslash u_{0}$ that has the greatest number of nodes (see Fig. 2. $\mathrm{c}$ ). If $\left|\alpha_{j}\right|+\left|T\left(l_{j}\right)\right| \leq\left|\beta_{j}\right|+\left|T\left(r_{j}\right)\right|$ then set $v_{j+1}=r_{j}$, otherwise set $v_{j+1}=l_{j}$. Similarly to [2], we get the following:

Lemma 1. For any left subtree $\alpha$ of $\mathcal{U} \backslash u_{0}$ or right subtree $\alpha$ of $\mathcal{V} \backslash v_{0}$ and for any right subtree $\beta$ of $\mathcal{U} \backslash u_{0}$ or left subtree $\beta$ of $\mathcal{V} \backslash v_{0},|\alpha|+|\beta| \leq n / 2$.

Proof: If $\alpha$ and $\beta$ are both subtrees of $\mathcal{U} \backslash u_{0}$ or if are both subtrees of $\mathcal{V} \backslash v_{0}$, then the statement follows as in Lemma 4.1 of [2. Otherwise, suppose $\alpha$ is a left subtree of $\mathcal{U} \backslash u_{0}$ and $\beta$ is a left subtree of $\mathcal{V} \backslash v_{0}$. Let $v_{j}$ be the parent of $\beta$ 's root. Denote by $l_{j}$ and $r_{j}$ the left and right child of $v_{j}$, respectively. Notice that $r_{j}=v_{j+1}$. Denote by $\alpha_{j}$ the one between the heaviest right subtree of path $\left(v_{1}, \ldots, v_{j-1}\right)$ and the heaviest left subtree of $\mathcal{U} \backslash u_{0}$ that has the greatest number of nodes, and denote by $\beta_{j}$ the one between the heaviest left subtree of path $\left(v_{1}, \ldots, v_{j-1}\right)$ and the heaviest right subtree of $\mathcal{U} \backslash u_{0}$ that has the greatest number of nodes. By construction $\left|\alpha_{j}\right|+\left|T\left(l_{j}\right)\right| \leq\left|\beta_{j}\right|+\left|T\left(r_{j}\right)\right|$. Moreover, $\left|\alpha_{j}\right|+\left|T\left(l_{j}\right)\right|+\left|\beta_{j}\right|+\left|T\left(r_{j}\right)\right| \leq n$. Therefore, $\alpha_{j}+\left|T\left(l_{j}\right)\right| \leq n / 2$. Since $\alpha \leq \alpha_{j}$ and $\beta=T\left(l_{j}\right)$, the statement follows. The case in which $\alpha$ is a right subtree of $\mathcal{V} \backslash v_{0}$ and $\beta$ is a right subtree of $\mathcal{U} \backslash u_{0}$ is analogous.

Selecting $\pi$ as just described, we get $W(n) \leq \max _{n_{1}+n_{2} \leq n / 2} W\left(n_{1}\right)+W\left(n_{2}\right)+1$. As already noticed in [2], by Hölder's inequality $n_{1}+n_{2} \leq n / 2$ implies $\sqrt{n_{1}}+$ $\sqrt{n_{2}} \leq \sqrt{n}$ and, by induction, $W(n) \leq c \sqrt{n}-1$, for some constant $c$ depending only on the values of $W(n)$ with $n$ small.

\section{Straight-Line Orthogonal Drawings of Ternary Trees}

In this section we consider SO-drawings of ternary trees. First, we show that if an order of the children of each node is fixed, then quadratic area is necessary in the worst case. 
Theorem 3. There exists an $n$-node ternary tree $T$ requiring $\Omega\left(n^{2}\right)$ area in any order-preserving SO-drawing.

Proof: Assume $n \equiv 4 \bmod$ 9. Tree $T$ is composed of (see Fig. 3. a): (i) a spine $C_{1}:\left(m_{0}=r(T), m_{1}, \ldots, m_{\frac{n-4}{9}}, m_{\frac{n+5}{9}}\right)$, with $m_{1}$ left child of $r(T)$ and $m_{i}$ middle child of $m_{i-1}$, for $i=2,3, \ldots, \frac{n+5}{9}$; (ii) a spine $C_{2}:\left(p_{0}=r(T), p_{1}, \ldots, p_{\frac{n-4}{9}}, p_{\frac{n+5}{9}}\right)$, with $p_{i}$ middle child of $p_{i-1}$, for $i=1,2, \ldots, \frac{n+5}{9}$; (iii) a spine $C_{3}:\left(q_{0}=\right.$ $r(T), q_{1}, \ldots, q_{\frac{n-4}{9}}, q_{\frac{n+5}{9}}$, with $q_{1}$ right child of $r(T)$ and $q_{i}$ middle child of $q_{i-1}$, for $i=2,3, \ldots, \frac{n+5}{9}$; and (iv) a left and a right child for each node $m_{i}, p_{i}$, and $q_{i}$, for $i=1,2, \ldots, \frac{n-4}{9}$. Consider any order-preserving SO-drawing of $C_{1}$ and of the children of nodes $m_{i}$, with $1 \leq i \leq \frac{n-4}{9}$. Suppose that $m_{1}$ is to the left of $m_{0}$. Then, to preserve the order of the children of $m_{1}, m_{i}$ is to the left of $m_{i-1}$, for $i=2,3, \ldots, \frac{n+5}{9}$. Analogously, if $m_{1}$ is to the right, above, or below $m_{0}$, then $m_{i}$ is to the right, above, or below $m_{i-1}$, respectively, for $i=2,3, \ldots, \frac{n+5}{9}$. Such an argument applies to $C_{2}$ (to $C_{3}$ ), as well: If $p_{1}\left(q_{1}\right)$ is to the left, to the right, above, or below $p_{0}\left(q_{0}\right)$, then $p_{i}\left(q_{i}\right)$ is to the left, to the right, above, or below $p_{i-1}\left(q_{i-1}\right)$, respectively, for $i=2,3, \ldots, \frac{n+5}{9}$. Since $r(T)$ has three children, then at least one of them is above or below $r(T)$ and one of them is to the left or to the right of $r(T)$. Hence, any order-preserving SO-drawing of $T$ has at least $\frac{n+5}{9}+1$ height and width.

The proved bound is tight, as shown in the following:

Theorem 4. Any n-node ternary tree $T$ admits an $O\left(n^{2}\right)$ area order-preserving SO-drawing.

Proof: We show an inductive algorithm constructing an order-preserving SOdrawing $\Gamma$ of $T$ satisfying the top visibility property. If $n=1$, then $\Gamma$ is trivially constructed. Suppose $n>1$. Let $T_{l}, T_{m}$, and $T_{r}$ be the left, middle, and right subtree of $r(T)$. By induction, drawings $\Gamma_{l}, \Gamma_{m}$, and $\Gamma_{r}$ satisfying the top visibility property can be constructed for $T_{l}, T_{m}$, and $T_{r}$, respectively. Draw $r(T)$ in the plane. Rotate $\Gamma_{l}$ of $\pi / 2$ in clockwise direction. Place $\Gamma_{l}$ with the rightmost vertical line intersecting it one unit to the left of $r(T)$ and with $r\left(T_{l}\right)$ on the same horizontal line of $r(T)$. Rotate $\Gamma_{r}$ of $\pi / 2$ in counter-clockwise direction. Place $\Gamma_{r}$ with the leftmost vertical line intersecting it one unit to the right of $r(T)$ and with $r\left(T_{r}\right)$ on the same horizontal line of $r(T)$. Place $\Gamma_{m}$ with the highest horizontal line intersecting it one unit below the lowest horizontal line intersecting $\Gamma_{l}$ or $\Gamma_{r}$ and with $r\left(T_{m}\right)$ on the same vertical line of $r(T)$ (see Fig. 3. b). It's easy to see that $\Gamma$ is an order-preserving SO-drawing satisfying the top visibility property. Since $\Gamma$ has at least one node for each horizontal and vertical grid line intersecting it, then its height and its width are $O(n)$.

For non-order-preserving drawings better bounds can be achieved:

Theorem 5. Any n-node ternary tree $T$ admits an $O\left(n^{1.631}\right)$ area $S O$-drawing.

Proof: We show an inductive algorithm that constructs an SO-drawing $\Gamma$ of $T$ satisfying the top visibility property. If $n=1$, then $\Gamma$ is trivially constructed. 


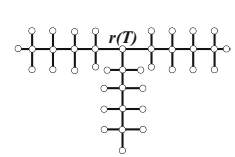

(a)

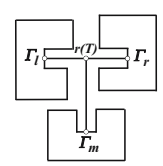

(b)

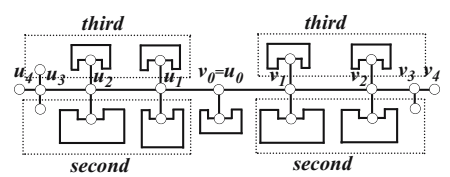

(c)

Fig. 3. (a) Tree $T$ requiring $\Omega\left(n^{2}\right)$ area in any order-preserving SO-drawing. (b) Illustration for the algorithm in Theorem 4 (c) Illustration for the algorithm in Theorem 5 Subtrees labeled by second or third are second or third heaviest subtrees, respectively.

Suppose $n>1$. Select a double-spine $\pi=\left(u_{k}, u_{k-1}, \ldots, u_{1}, u_{0}=r(T)=\right.$ $\left.v_{0}, v_{1}, \ldots, v_{m}\right)$ in $T$ such that: $T\left(v_{1}\right)$ is the heaviest subtree of $r(T)$; for $j=$ $2,3, \ldots, m, T\left(v_{j}\right)$ is the heaviest subtree of $v_{j-1} ; T\left(u_{1}\right)$ is the heaviest subtree of $r(T)$ different from $T\left(v_{1}\right)$; for $i=2,3, \ldots, k, T\left(u_{i}\right)$ is the heaviest subtree of $u_{i-1}$. For each node $v_{j}$ in $\pi$, with $j=0,1, \ldots, m-1$, (for each node $u_{i}$ in $\pi$, with $i=1,2, \ldots, k-1)$, call second heaviest subtree $S\left(v_{j}\right)\left(S\left(u_{i}\right)\right)$ and third heaviest subtree $R\left(v_{j}\right)\left(R\left(u_{i}\right)\right.$ ), the subtrees of $v_{j}$ (of $u_{i}$ ) different from $T\left(v_{j+1}\right)$ (from $\left.T\left(u_{i+1}\right)\right)$ with the greater and the smaller number of nodes, respectively.

Recursively construct a drawing satisfying the top visibility property of each subtree of $\pi$. Let $h$ be an horizontal grid line. Draw $r(T)$ on $h$. Place the drawing of $R\left(v_{0}\right)$ with the highest horizontal line intersecting it one unit below $h$ and with its root on the same vertical line of $r(T)$. For $j=1,2, \ldots, m-1$, rotate of $\pi$ the drawing of $R\left(v_{j}\right)$. Place the drawing $\Gamma_{j}^{S}$ of $S\left(v_{j}\right)$ and $\Gamma_{j}^{R}$ of $R\left(v_{j}\right)$ so that the highest horizontal line intersecting $\Gamma_{j}^{S}$ is one unit below $h$, the lowest horizontal line intersecting $\Gamma_{j}^{R}$ is one unit above $h$, their roots are on the same vertical line, and the leftmost vertical line intersecting $\Gamma_{j}^{S}$ or $\Gamma_{j}^{R}$ is one unit to the right of the rightmost vertical line intersecting $\Gamma_{j-1}^{S}, \Gamma_{j-1}^{R}$, or $v_{j-1}$. Draw $v_{j}$ on $h$ on the same vertical line of its already drawn children (or draw $v_{j}$ one unit to the right of the rightmost vertical line intersecting $\Gamma_{j-1}^{S}, \Gamma_{j-1}^{R}$, or $v_{j-1}$ if no child of $v_{j}$ has been drawn). Draw $v_{m}$ on $h$ one unit to the right of the rightmost vertical line intersecting $\Gamma_{m-1}^{S}, \Gamma_{m-1}^{R}$, or $v_{m-1}$. For path $\left(u_{1}, u_{2}, \ldots, u_{k}\right)$ and its subtrees, analogously construct a drawing in which the path lies on $h$, to the left of $r(T)$, and the $S\left(u_{i}\right)$ 's and the $R\left(u_{i}\right)$ 's are below and above $h$, respectively (see Fig. 3. c).

It's easy to see that $\Gamma$ is an SO-drawing satisfying the top visibility property. Let's analyze the area of $\Gamma$. Since there is at least one node of $T$ for each vertical grid line intersecting $\Gamma$, then its width is $O(n)$. Denote by $H(T)$ the height of the drawing constructed by the algorithm when its input is $T$. Let also $H(n)=\max \{H(T)\}$ over all ternary trees $T$ with $n$ nodes. Since all subtrees drawn above $\pi$ (below $\pi$ ) are aligned on their bottom side (on their top side) and since $H(n)$ is a non-decreasing function of $n$, then $H(n)=H\left(n_{a}\right)+H\left(n_{b}\right)+1$, where $n_{a}\left(n_{b}\right)$ is the number of nodes in the heaviest subtree drawn above (below) of $\pi$. We claim (1) $n_{a}+n_{b} \leq 2 n / 3$. Namely, we have (2) $n_{a} \leq n_{b}$, (3) $n_{b} \leq n / 2$, and (4) $n_{a} \leq n-2 n_{b}$. Inequality (2) holds since for each node $w$ in $\pi,|R(w)| \leq$ $|S(w)|$; inequality (3) follows from the fact that, for each node $v_{j}$ and $u_{i}$ in $\pi$, 
$\left|S\left(v_{j}\right)\right| \leq\left|T\left(v_{j+1}\right)\right|$ and $\left|S\left(u_{i}\right)\right| \leq\left|T\left(u_{i+1}\right)\right|$; inequality (4) follows by considering any node $v_{j}\left(u_{i}\right)$ in $\pi$ and observing that $\left|S\left(v_{j}\right)\right| \leq\left|T\left(v_{j+1}\right)\right|\left(\left|S\left(u_{i}\right)\right| \leq\left|T\left(u_{i+1}\right)\right|\right)$ and that $\left|R\left(v_{j}\right)\right|+\left|S\left(v_{j}\right)\right|+\left|T\left(v_{j+1}\right)\right| \leq n\left(\left|R\left(u_{i}\right)\right|+\left|S\left(u_{i}\right)\right|+\left|T\left(u_{i+1}\right)\right| \leq n\right)$. By (3) we have $n_{b}=\frac{n}{2}-\alpha$, with $\alpha \geq 0$. If $\alpha \geq n / 6$, then by (2) $n_{b}+n_{a} \leq$ $2(n / 2-\alpha) \leq 2 n / 3$. If $\alpha<n / 6$, by (4) we have $n_{a} \leq n-2(n / 2-\alpha)=2 \alpha$. Hence $n_{b}+n_{a} \leq n / 2-\alpha+2 \alpha=n / 2+\alpha \leq 2 n / 3$. We claim that $n_{a}^{\left(1 / \log _{2} 3\right)}+n_{b}^{\left(1 / \log _{2} 3\right)} \leq$ $n^{\left(1 / \log _{2} 3\right)}$. Hölder's inequality states that $(5) \sum a_{i} b_{i} \leq\left(\sum a_{i}^{p}\right)^{\frac{1}{p}}\left(\sum b_{i}^{q}\right)^{\frac{1}{q}}$ for every $p$ and $q$ such that $1 / p+1 / q=1$. Substituting into (5) the values $a_{1}=n_{a}^{\left(1 / \log _{2} 3\right)}$, $a_{2}=n_{b}^{\left(1 / \log _{2} 3\right)}, b_{1}=b_{2}=1,1 / p=1 / \log _{2} 3$, and $1 / q=1-1 / \log _{2} 3$, we get $n_{a}^{\left(1 / \log _{2} 3\right)}+n_{b}^{\left(1 / \log _{2} 3\right)} \leq\left[\left(n_{a}^{\left(1 / \log _{2} 3\right)}\right)^{\log _{2} 3}+\left(n_{b}^{\left(1 / \log _{2} 3\right)}\right)^{\log _{2} 3}\right]^{\left(1 / \log _{2} 3\right)} \cdot[1+$ $1]^{\left(1-1 / \log _{2} 3\right)}=\left(n_{a}+n_{b}\right)^{\left(1 / \log _{2} 3\right)} \cdot 2^{\left(1-1 / \log _{2} 3\right)} \leq(2 n / 3)^{\left(1 / \log _{2} 3\right)} \cdot 2^{\left(1-1 / \log _{2} 3\right)}=$ $n^{\left(1 / \log _{2} 3\right)}\left(2^{1 / \log _{2} 3} \cdot 2 \cdot 2^{-1 / \log _{2} 3}\right) /\left(3^{1 / \log _{2} 3}\right)=n^{\left(1 / \log _{2} 3\right)}\left(2 /\left(3^{1 / \log _{2} 3}\right)\right)=$ $n^{\left(1 / \log _{2} 3\right)}$. Hence, $H(n) \leq \max _{n_{1}+n_{2} \leq 2 n / 3} H\left(n_{1}\right)+H\left(n_{2}\right)+1$ by induction solves to $H(n)=c \cdot n^{\left(1 / \log _{2} 3\right)}-1$ for some constant $c$, depending only on the values of $H(n)$ with $n$ small. It follows that $H(n)=O\left(n^{\left(1 / \log _{2} 3\right)}\right)=O\left(n^{0.631}\right)$.

\section{Straight-Line Orthogonal Drawings of Complete Ternary Trees}

For complete ternary trees we present two algorithms constructing drawings with better area bounds than the ones obtained for general ternary trees. Let $\Gamma_{h}$ be a drawing of a complete ternary tree $T_{h}$ with height $h$. In both algorithms inductively suppose to have a drawing $\Gamma_{h-1}$ of $T_{h-1}$ satisfying the top visibility property, take three copies $\Gamma_{h-1}^{\prime}, \Gamma_{h-1}^{\prime \prime}$, and $\Gamma_{h-1}^{\prime \prime \prime}$ of $\Gamma_{h-1}$, rotate $\Gamma_{h-1}^{\prime}$ of $\pi / 2$ and $\Gamma_{h-1}^{\prime \prime}$ of $3 \pi / 2$ in clockwise direction. The algorithms differ in the geometric construction of $\Gamma_{h}$. In Construction 1 draw $r\left(T_{h}\right)$ on any horizontal grid line $l_{h}$. Place $\Gamma_{h-1}^{\prime \prime \prime}$ with the highest horizontal line intersecting it one unit below $l_{h}$ and with the root $r\left(T_{h-1}\right)$ in $\Gamma_{h-1}^{\prime \prime \prime}$ on the same vertical line of $r\left(T_{h}\right)$. Place $\Gamma_{h-1}^{\prime}$ with the rightmost vertical line intersecting it one unit to the left of the leftmost vertical line intersecting $\Gamma_{h-1}^{\prime \prime \prime}$ and with the root $r\left(T_{h-1}\right)$ in $\Gamma_{h-1}^{\prime}$ on $l_{h}$. Place $\Gamma_{h-1}^{\prime \prime}$ with the leftmost vertical line intersecting it one unit to the right of the rightmost vertical line intersecting $\Gamma_{h-1}^{\prime \prime \prime}$ and with the root $r\left(T_{h-1}\right)$ in $\Gamma_{h-1}^{\prime \prime}$ on $l_{h}$ (see Fig. 4a). In Construction 2 draw $r\left(T_{h}\right)$ on any horizontal grid line $l_{h}$. Place $\Gamma_{h-1}^{\prime}$ with the rightmost vertical line intersecting it one unit to the left of $r\left(T_{h}\right)$ and with the root $r\left(T_{h-1}\right)$ in $\Gamma_{h-1}^{\prime}$ on $l_{h}$. Place $\Gamma_{h-1}^{\prime \prime}$ with the leftmost vertical line intersecting it one unit to the right of $r\left(T_{h}\right)$ and with the root $r\left(T_{h-1}\right)$ in $\Gamma_{h-1}^{\prime \prime}$ on $l_{h}$. Place $\Gamma_{h-1}^{\prime \prime \prime}$ with the highest horizontal line intersecting it one unit below the lowest horizontal line intersecting $\Gamma_{h-1}^{\prime \prime}$, and with the root $r\left(T_{h-1}\right)$ in $\Gamma_{h-1}^{\prime \prime \prime}$ on the same vertical line of $r\left(T_{h}\right)$ (see Fig. 4. b). We have the following:

Theorem 6. An n-node complete ternary tree $T_{h}$ admits an $O\left(n^{1 / \log _{4} 3}\right)=$ $O\left(n^{1.262}\right)$ area SO-drawing. 


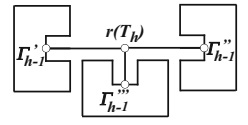

(a)

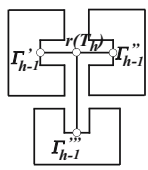

(b)

Fig. 4. Constructions 1 (a) and 2 (b) for SO-drawings of complete ternary trees

Proof: Construct a drawing $\Gamma_{h}$ of $T_{h}$ by inductively using Construction 1. Denoting by $W_{h}$ and by $H_{h}$ the width and the height of $\Gamma_{h}$, respectively, by construction we have $W_{h}=W_{h-1}+2 H_{h-1}$ and $H_{h}=\max \left\{W_{h-1}, H_{h-1}+\left(W_{h-1}+\right.\right.$ 1) $/ 2\}$. Assume by inductive hypothesis that $W_{h-1}=2^{h-1}-1$ and that $H_{h-1}=$ $2^{h-2}$. Notice that this holds in the base case, where $W_{1}=H_{1}=1$. Observe also that by inductive hypothesis $H_{h-1}+\left(W_{h-1}+1\right) / 2=2^{h-2}+\left(2^{h-1}-1+1\right) / 2=$ $2^{h-1}>W_{h-1}=2^{h-1}-1$. Hence, $H_{h}=2^{h-1}$ and $W_{h}=2^{h-1}-1+2 \cdot 2^{h-2}=2^{h}-1$, that proves the inductive hypothesis. The area of $\Gamma_{h}$ is equal to $\left(2^{h}-1\right) \cdot 2^{h-1}<$ $4^{h}=4^{O\left(\log _{3} n\right)}=O\left(n^{1 / \log _{4} 3}\right)$. Inductively applying Construction 2 instead of Construction 1 yields to a drawing with asymptotically the same area.

Next, we show that $n$-node complete ternary trees have $\Omega\left(n^{0.438}\right)$ minimum side in any SO-drawing. This result sharply contrasts with the analogous for binary trees. Namely, any binary tree admits an SO-drawing in which one side is $O(\log n)$. Let $\Gamma_{h}$ be any SO-drawing of $T_{h}$. One of the children of $r\left(T_{h}\right)$, say $v_{1}$, is such that no other child of $r\left(T_{h}\right)$ is drawn on the line $l$ through $r\left(T_{h}\right)$ and $v_{1}$. Moreover, for $i=1,2, \ldots, h-2$, node $v_{i}$ has exactly one child $v_{i+1}$ drawn on $l$. Hence, in any SO-drawing of $T_{h}$, there is a spine of $h$ nodes drawn all on the same horizontal or vertical line $l$, such that no other child of $r\left(T_{h}\right)$ is on $l$. We call leg of $\Gamma_{h}$ such a spine. Analogously, in any SO-drawing of $T_{h}$ there is a double-spine of $2 h-1$ nodes that are drawn all on the same horizontal or vertical line. We call arm of $\Gamma_{h}$ such a double-spine. We have:

Lemma 2. The minimum side of any $S O$-drawing of an $n$-node complete ternary tree is $\Omega\left(n^{0.438}\right)$.

Proof: Let $\Gamma_{h}$ be an SO-drawing of a complete ternary tree $T_{h}$ in which the length of the leg is minimum. Let $l\left(\Gamma_{h}\right)$ be the length of the leg in $\Gamma_{h}$. We claim that $l\left(\Gamma_{h}\right) \geq l\left(\Gamma_{h-1}\right)+l\left(\Gamma_{h-2}\right)$. Consider the arms of the subtrees of $r\left(T_{h}\right)$. Either two of such arms are vertical and one horizontal or vice versa. Assume,

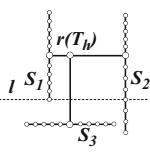

(a)

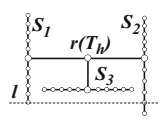

(b)

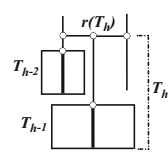

(c)

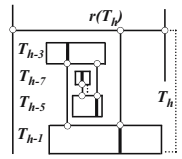

(d)

Fig. 5. Illustrations for Lemma 2 Thick lines drawn inside subtrees represent their legs. 
possibly rotating $\Gamma_{h}$ of $\pi / 2$, that two of such arms, say $S_{1}$ and $S_{2}$, are vertical and one, say $S_{3}$, horizontal. Consider the possible non-crossing placements of $S_{1}, S_{2}$, and $S_{3}$, and consider the lowest horizontal line $l$ intersecting both $S_{1}$ and $S_{2}$. Two are the cases; either $S_{3}$ is below $l$ (Fig. 5] a), or not (Fig. [5.b). In the first case we trivially have $l\left(\Gamma_{h}\right)>l\left(\Gamma_{h-1}\right)+l\left(\Gamma_{h-2}\right)$ (see Fig. 5 c) and the claim follows. In the second case we have $l\left(\Gamma_{h}\right)>l\left(\Gamma_{h-1}\right)+l\left(\Gamma_{h-3}\right)+l\left(\Gamma_{h-5}\right)+$ $\ldots+l\left(\Gamma_{h / 2-\lfloor h / 2\rfloor+3}\right)+l\left(\Gamma_{h / 2-\lfloor h / 2\rfloor+1}\right)$ (see Fig. 5.d). However, recurrence (1) $f(x)=f(x-1)+f(x-3)+f(x-5)+\ldots+f(x / 2-\lfloor x / 2\rfloor+3)+f(x / 2-\lfloor x / 2\rfloor+1)$ asymptotically provides for $f(x)$ the same value provided by $f(x)=f(x-1)+$ $f(x-2)$. Namely, from (1) we get $f(x-2)=f(x-3)+f(x-5)+\ldots+$ $f((x-2) / 2-\lfloor(x-2) / 2\rfloor+3)+f((x-2) / 2-\lfloor(x-2) / 2\rfloor+1)$, and since $(x-2) / 2-\lfloor(x-2) / 2\rfloor=x / 2-\lfloor x / 2\rfloor$ we get $f(x-3)+f(x-5)+\ldots+$ $f(x / 2-\lfloor x / 2\rfloor+3)+f(x / 2-\lfloor x / 2\rfloor+1)=f(x-2)$, that substituted in (1) gives $f(x)=f(x-1)+f(x-2)$. Hence, $l\left(\Gamma_{h}\right) \geq l\left(\Gamma_{h-1}\right)+l\left(\Gamma_{h-2}\right)$, implying that $l\left(\Gamma_{h}\right)$ grows at least as the terms of the Fibonacci series, for which it is well know that the ratio of two consecutive terms $l_{k+1}$ and $l_{k}$ tends to the golden ratio $\phi$. Hence $l\left(\Gamma_{h}\right)=\Omega\left(\phi^{h}\right)=\Omega\left(\phi^{\log _{3} n}\right)=\Omega\left(n^{1 / \log _{\phi} 3}\right)=\Omega\left(n^{0.438}\right)$. The statement follows by observing that the minimum length of the arm of $\Gamma_{h}$ grows asymptotically at least as the leg of $\Gamma_{h}$ and that each side of $\Gamma_{h}$ is at least long as the leg or as the arm of $\Gamma_{h}$.

In the following we prove that, for complete ternary trees, the lower bound of Lemma 2 is tight. Again, we introduce two constructions, called Constructions $\hat{1}$ and $\hat{2}$, defined as follows: Construction $\hat{1}$ has the same geometric inductive step of Construction 1, but the side drawings are recursively constructed with Construction $\hat{2}$ and the base drawing is recursively constructed with Construction $\hat{1}$; Construction $\hat{2}$ has the same geometric inductive step of Construction 2, but the side drawings are recursively constructed with Construction $\hat{1}$ and the base drawing is recursively constructed with Construction $\hat{2}$.

Lemma 3. The drawings built by Construction $\hat{1}$ have $O\left(n^{0.438}\right)$ height.

Proof: Denote by $H_{h}^{1}$ (by $W_{h}^{2}$ ) the height (the width) of the drawing of a complete ternary tree with height $h$ built with Construction $\hat{1}$ (with Construction $\hat{2}$ ). By simple geometric considerations, we have (1) $H_{h}^{1}=\max \left\{W_{h-1}^{2}, H_{h-1}^{1}+\right.$ $\left.\left(W_{h-1}^{2}+1\right) / 2\right\}$ and $(2) W_{h}^{2}=\max \left\{W_{h-1}^{2}, 2 H_{h-1}^{1}+1\right\}$. Suppose by induction that $H_{h-1}^{1}+\left(W_{h-1}^{2}+1\right) / 2 \geq W_{h-1}^{2}$ and that $2 H_{h-1}^{1}+1 \geq W_{h-1}^{2}$. Such inductive hypotheses are verified in the base case, where $H_{1}^{1}=1$ and $W_{1}^{2}=1$. Due to the inductive hypotheses, (1) and (2) turn in (1') $H_{h}^{1}=H_{h-1}^{1}+\left(W_{h-1}^{2}+1\right) / 2$, and (2') $W_{h}^{2}=2 H_{h-1}^{1}+1$, respectively. We have $H_{h}^{1}+\left(W_{h}^{2}+1\right) / 2=H_{h-1}^{1}+\left(W_{h-1}^{2}+\right.$ $1) / 2+\left(2 H_{h-1}^{1}+1+1\right) / 2=2 H_{h-1}^{1}+\left(W_{h-1}^{2}\right) / 2+3 / 2>2 H_{h-1}^{1}+1=W_{h}^{2}$, and that $2 H_{h}^{1}+1=2 H_{h-1}^{1}+W_{h-1}^{2}+1+1>2 H_{h-1}^{1}+1=W_{h}^{2}$. Hence, the inductive hypothesis is verified and (1') and (2') hold. Substituting (2') into (1'), we get $H_{h}^{1}=H_{h-1}^{1}+\left(\left(2 H_{h-2}^{1}+1\right)+1\right) / 2=H_{h-1}^{1}+H_{h-2}^{1}+1$. As in the proof of Lemma 2, $H_{h}^{1}$ grows as the terms of the Fibonacci series, yielding $H_{h}^{1}=O\left(n^{0.438}\right)$. 


\section{Conclusions and Open Problems}

In this paper we have shown some upper bounds (Theorems 2, 4, 5, and 6) and lower bounds (Theorems 1 and 3) concerning the area requirement of straightline orthogonal drawings of binary and ternary trees. As can be noticed from Table 1 some of these bounds are asymptotically tight, whereas for others there is still space for improvements. In particular, for order-preserving SO-drawings of binary trees and for SO-drawings of ternary trees there are wide gaps between the area upper bounds we provided and the actual lower bounds. For complete ternary trees we conjecture that an algorithm combining Constructions 1 and 2 could improve the upper bound we provided here. However, the most fascinating problem in this area still remains, in our opinion, the one of determining whether binary trees admit straight-line orthogonal drawings in linear area.

\section{References}

1. Chan, T.M., Goodrich, M.T., Rao Kosaraju, S., Tamassia, R.: Optimizing area and aspect ratio in straight-line orthogonal tree drawings. Comput. Geom. 23(2), 153-162 (2002)

2. Chan, T.M.: A near-linear area bound for drawing binary trees. Algorithmica 34(1), 1-13 (2002)

3. Crescenzi, P., Di Battista, G., Piperno, A.: A note on optimal area algorithms for upward drawings of binary trees. Comput. Geom. 2, 187-200 (1992)

4. Di Battista, G., Eades, P., Tamassia, R., Tollis, I.G.: Graph Drawing. Prentice Hall, Upper Saddle River, NJ (1999)

5. Dolev, D., Trickey, H.W.: On linear area embedding of planar graphs. Technical report, Stanford, USA (1981)

6. Garg, A., Goodrich, M.T., Tamassia, R.: Planar upward tree drawings with optimal area. Int. J. Comput. Geometry Appl. 6(3), 333-356 (1996)

7. Garg, A., Rusu, A.: Area-efficient order-preserving planar straight-line drawings of ordered trees. Int. J. Comput. Geometry Appl. 13(6), 487-505 (2003)

8. Garg, A., Rusu, A.: Straight-line drawings of general trees with linear area and arbitrary aspect ratio. In: ICCSA, vol. (3), pp. 876-885 (2003)

9. Kim, S.K.: Simple algorithms for orthogonal upward drawings of binary and ternary trees sung. In: CCCG, pp. 115-120 (1995)

10. Shiloach, Y.: Arrangements of Planar Graphs on the Planar Lattice. PhD thesis, Weizmann Institute for Science (1976)

11. Shin, C.S., Kim, S.K., Chwa, K.Y.: Area-efficient algorithms for straight-line tree drawings. Comput. Geom. 15(4), 175-202 (2000)

12. Valiant, L.G.: Universality considerations in VLSI circuits. IEEE Trans. Comp. 30(2), 135-140 (1981) 